\title{
Subject Index Vol. 67, 1995
}

Abnormal development 132 Acidosis 370 Adrenal function 274 Adrenergic receptors 352

Adrenocorticotropic hormone

receptors 274 Adults 109 Alveolar gases 322 Amino acid oxidation 149 Amniotic cavity 100

- $\quad$ fluid 397

Analgesic, labor 140

Anoxia 370

Anticonvulsants 223

L-Arginine 240

Arterial pressure 432

Artery end-diastolic flow, absent

umbilical 21 Asphyxia 317,407

Barbiturates 432 Bilirubin 182,203 Blood flow 208 Blood-brain barrier 59 Body composition

414 Bottle feeding 94 Brain 203, 425

- $\quad$ development 64, 287

Brainstem 287

Breast feeding 94 Breathing, control 352 Bronchopulmonary dysplasia 54

Cardiorespiratory measurements

352 Catecholamine 287 Cell proliferation 317 Cerebral blood flow 425

metabolism 425

response 432 Cerebrospinal fluid 407 Chan Su 376

Chick embryo 441 Chicks, broiler 381 Chloramphenicol 230 Cholinesterases, 'faint-positive' 244

Choroid 208

Chromosomal nondisjunction 132

Chromosome analysis 132

Circadian rhythms 287

$\mathrm{CO} 2$ production rate 340

Cocaine 432

Cognition 140

Colliculi 287

Complement receptor 26

Containment 54

Control of respiration 322

Cooperation 387

Corticosterone 381

Cortisol 274

Cortisone acetate 113

Delivery 140

Development 77 
Diabetes mellitus 132

Dietary carbohydrate restriction

122 Differentiation 77 1 $\alpha, 25$-Dihydroxyvitamin D3 194 Dipeptidases 34 DNA 360 Dopamine 287 Doppler ultrasound 346 Doppler velocimetry 21 Drug abuse 432 Duodenal aspirate 248

Elastase 59

Embryogenesis 77

Encephalopathy, bilirubin 203

Endothelin 216

Endothelins, plasma concentrations 317

End-tidal carbon monoxide, neonates 182

Enteroglucagon 47

Ethanol 432

Fetal distress 21

growth 186

lactate uptake 295

liver 295

lung development 264

metabolism 149

rat hepatocytes 194 Fetoscopy 47

Fetus 47,154,230,397 Flow-through system 340 Folate 154 Forestomach 113

Gastric inhibitory polypeptide 47

Gastrin 47

Gastroschisis 244

Gel densitometry 244

Gene expression 77

Gentamicin, loading dose 13

Glandular stomach 113

Glomerular microcirculation 216

Glucagon 47

Glutathione S-transferase 230

Granulocytes 26

Group I phospholipase A2 113

- $\quad$ II phospholipase A2 113

Hammock 54 Hemodynamics 208 Holding 54 Human 397

- $\quad$ newborns 84

Hydroxyproline 34

5-Hydroxytryptamine 122

Hyperoxemia 208

Hypersensitivity 223

Hypothyroxinemia 64

Hypoxemia 208,216,352

Hypoxia 317,425

Hypoxic-ischemic encephalopathy

407

Ileum 113 
Immature kidney 216 Immunoglobulin receptor 26 Infants 414 Insulin 47, 186,441 Intestinal absorption 254

- $\quad$ epithelial cells 330

Intestine 360

Intravenous immunoglobulins 450 Intraventricular hemorrhage 317

455

Iodine deficiency 64 Iron saturation 330

Kernicterus 203 Ketone body flux 84 Kinetics 203

Lactateflux 295 Lactoferrin 330 Lecithin 34 Leukotrienes 425 Lipase activity 248 Lipid 381

A 109 Lipoprotein 381 Lipoxygenase 425 Liver 360

development 387 Liver-specific transcription

factors 387 Low birth weight 39, 160

Macaca mulatta 140 Macromolecular transport 254 Mammals 387 Meconium 100 Medulla 287

Meperidine 140 Microspheres 425 Middle cerebral artery 21 Monoamines 407 Motilin 47

Myeloperoxidase 167

Necrotizing enterocolitis 172 Neonatal adaptation 397

bladder 370

metabolism 149

rats 122 Neonate(s) 109,397 -, preterm 317 Neural tube defect 154 Neurodevelopmental

impairment

39 Neurotransmitter 287 Neutrophil 167 Newborn 450

infant(s) 94, 322 Nitric oxide 72,240

- synthase 240 Normal newborn infants,

ethnicity 182 Nutrition 172

Obstetric factors 39 Olfaction 397 Ontogeny 113 Operant testing 140 Opiate 140 Organ growth

360 Ornithine decarboxylase 441 Oxygen 208

- $\quad$ saturation 54,94

Pancreatic polypeptide 47

Patent ductus arteriosus 167

Pentoxifylline 72

Perinatal outcome 21

Peripheral chemoreceptors 352

Persistent pulmonary hypertension, newborn 240

Phagocytes 26

Phosphatidylcholine 264

Pig(s) 360

-, neonatal 274

Piglets 59

Placental lactate 295

Pons 287

Portable device 340

Postimplantation stage embryos 132

Pregnancy 376

Premature infant(s) 167

-, growth 172

newborns 84 Prenatal diagnosis 34,154 
perception 397

Preterm infant(s) 248, 340, 346 Prolidase 34 Protein 360

kinaseA 441

- C 441 Proteinase inhibitor 59

Receptor-mediated transcytosis

254 Red blood cells 109 Regional uptake 203 Renal blood flow velocity 346 Reoxygenation 208

Respiratory control 287

distress syndrome $167,223,317$

-- , critically ill premature

infants 172

- $\quad$ pattern 322

Retina 208

Retinoicacid 264 Retinoid acid receptor 264 Retinol 264

- $\quad$ deficiency 264

Retinyl palmitate 264

Reverse transcriptase-polymerase

chain reaction 264 Rhesus monkey infant 140 Risk factors, obstetric 160 RNA 360

Septic shock 72

Serotonin 100

Smooth muscle contractility 370

Spontaneous behavior 140

Stem cells 77

Streptozotocin 186

T3 receptor 64

Teratoma 244

Thrombocytopenia 450

Tissue distribution 295

Toad glandular secretion 376

Total body water 414

Total-body electrical conductivity 414

Toxicity 376

Trypsin activity 248

Tryptophan 122

Tumor necrosis factor- $\alpha 72$

Tyrosine kinase, insulin-dependent 441

Umbilical lactate uptake 295

- $\quad$ venous perfusion 100

United Arab Emirates, incidence

160 Urea excretion rate 149

production rate 149

Vasoactive factors 216 Vasoconstriction 100,317 Vasomotor nephropathy 216 VCO2

measurements 340 Ventilation 322 Vitamin D metabolism 194

- $\quad$ - receptors 194

Yersinia enterocolitica 360 
Subject Index 